\title{
STERIC EFFECTS IN THE MASS SPECTRA OF THE STEREOISOMERS OF DECALIN-1,3-DIOL AND OF 1,3-DIMETHOXY-DECALIN $\dagger$
}

\author{
HANS-Fr. GRÜTZMAChER* and GUNTER TOLKIEN \\ Fakultät für Chemie der Universität Bielefeld, 4800 Bielefeld, Postfach 8640 . Germany
}

(Received in the UK I June 1976; Accepted for publication 3 August 1976)

\begin{abstract}
The stereoisomers 1a-8a of decalin-1,3-diol have been synthesized by LAH-reduction of cis - and transdecalin-1,3-dione, respectively. With the exception of trans-decalin-la,3e- and -le,3a-diols, 7a and 8a, the stereoisomers have been isolated by column chromatography, and their configurations have been determined by 'H-NMR, IR and chemical methods. It is shown by the aid of deuterated derivatives, that the elimination of $\mathrm{H}_{2} \mathrm{O}$, $\mathrm{MeOH}$ and $\mathrm{CH}_{2} \mathrm{O}$ from the molecular ions of the stereoisomeric diols and di-O-methyl ethers, respectively, occurs predominantly by stereospecific reactions, if the ground state conformation of the molecule corresponds to the geometry of the transition state of the elimination reaction. The steric control of the fragmentations is greatly reduced, if conformational changes of the molecular ions have to occur prior to fragmentation. No clear steric effects are observed, if none of the conformations of the intact molecular ions corresponds to the transition state. These steric effects can be used to identify the various stereoisomers of decalin-1,3-diol and 1,3-dimethoxy-decalin by mass spectrometry.
\end{abstract}

In continuation of our studies on the steric effects in the mass spectra of cyclic and bicyclic polyol derivatives, ${ }^{1}$ we synthesized the stereoisomers $1-\mathbf{8}$ of decalin-1,3-diol and investigated their mass spectrometric fragmentation. It is known, that large steric effects occur in the elimination of HOR $(R=H, M e)$ from the molecular ions of cyclic diol derivatives and that the intensities of the corresponding fragment ions in the EI mass spectra, obtained by the usual conditions of analytical mass spectrometry, can be used for an identification of stereoisomers of cyclohexane-diol-derivatives. ${ }^{2}$ In a previous publication it was shown, that similar steric effects are observed in the mass spectra of stereoisomeric decalin-1,4-diols and their $\mathrm{O}$-Me derivatives. ${ }^{3}$ Analogous mass spectrometric behaviour of cyclohexane-1,3-diols and of decalin-1,3-diols, respectively would therefore be expected. However, it has recently been observed, that the conformational mobility of the molecular ions has an influence on the stereochemical control of the elimination reactions. ${ }^{4.5}$ Since the conformational mobility of the decalins is reduced, due to the presence of a second ring, it was of interest to see, whether steric effects are still observed in the mass spectra of decalin-1,3-diol derivatives and whether mass spectrometry can be used as an analytical tool for the identification of stereoisomers in this class of compounds.

Synthesis and identification of compounds. The 8 stereoisomers of decalin-1,3-diol have not been described in the literature. The usual method of synthesis of cyclic alcohols is catalytic hydrogenation of the corresponding phenols. However, the mixture of aliphatic diols obtained from 1,3-dihydroxynaphthalene consisted mostly of the cis - decalin - 1,3 - diols 1a-4a and only a small amount of diols 5a-8a derived from trans-decalin had been formed. Therefore we chose reduction of cis - and trans - decalin 1,3 - dione, respectively, by $\mathrm{LAH}$ to synthesize the 1,3-diols with the skeleton of cis-and trans-decalin. The

† Mechanisms of Mass Spectrometric Fragmentation Reaction-XVII. Part XVI: U. Neuert and H. F. Grützmacher, Org. Mass Spectrom. 11, (1976) in press. same method, using $\mathrm{LAD}$ instead of $\mathrm{LAH}$, gave the deuterated compounds 1c-8c. No interchanges between the cis- and trans-decalin systems was observed. The decalin-1,3-diones ${ }^{6}$ were obtained from 1 - acetyl cyclohexene and sodium diethyl malonate via 4 carbethoxy - cis - decalin - 1,3 - dione after hydrolysis and decarboxylation (Scheme 1).

The mixture of cis - decalin - 1.3 - diols obtained by reduction of the corresponding dione was analyzed by gas chromatography of the bis-trifluoracetates (Table 1). One isomer, 3a, was formed in large amounts, while isomer $4 \mathbf{a}$ was only a minor component of the mixture. Compounds la and 4a were isolated as pure compounds by repeated column chromatography (silica gel, acetone/benzene, $2: 3$, $v / v)$, but, 2a and 3a were eluted as a mixture, from which pure 3a was obtained by recrystallization from benzene. We did not succeed in isolating pure $2 \mathbf{a}$, therefore the mixture composed of $48 \%$ 2a and $52 \%$ 3a (by gas chromatography) was used for the mass spectrometric investigations. As the mass spectrum of pure $3 \mathbf{a}$ and the composition of the mixture was known, the mass spectrum of $2 \mathrm{a}$ could be calculated from that of the mixture by standard methods of quantitative mass spectrometric analysis of mixtures.

Similarly the mixture of diols obtained from trans decalin - 1,3 - dione by reduction with LAH was analyzed by gas chromatography (Table 1). Only three peaks were observed in the gas chromatogram. and as was shown later by the 'H-NMR and IR spectra, the peak with the retention time of $24.55 \mathrm{~min}$ was due to the bis-trifluoracetates of $7 \mathbf{a}$ and $8 \mathbf{a}$. It was not possible to separate and isolate 7a and 8a, therefore a mixture of these isomers had to be used for the subsequent investigations. Isomers 5a and $6 \mathrm{a}$ were obtained as pure substances by repeated column chromatography (silica gel, benzene/acetone $3: 1$ $\mathrm{v} / \mathrm{v}$ ) of the bis-trimethylsilyl ethers of the mixture of the trans-diols and subsequent hydrolysis by boiling with aqueous methanol.

The stereochemistry of compounds 1a-8a was determined by a combination of physical and chemical methods. The configuration of the carbon skeleton was 


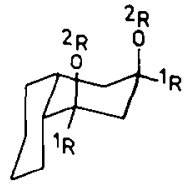

$\underline{\underline{1}}$

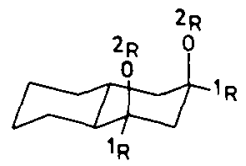

$\underline{5}$

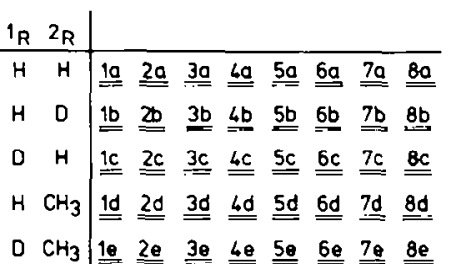

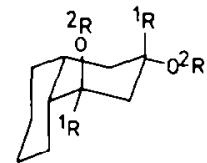

$\stackrel{3}{=}$

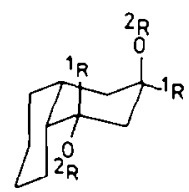

$\underline{-}$

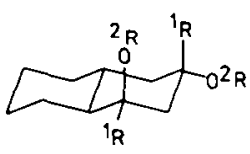

2

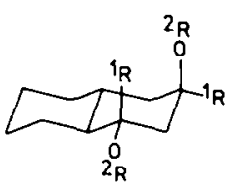

$\stackrel{8}{=}$

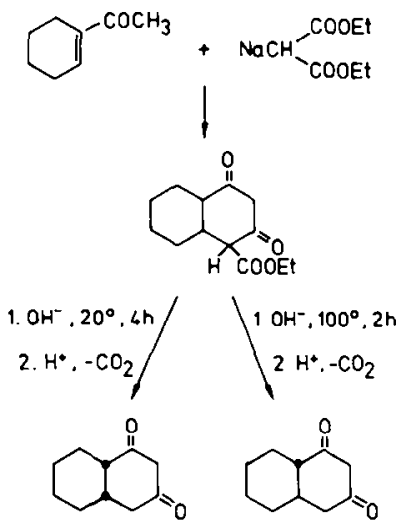

Scheme 1 .

Table 1. Gas chromatographic analysis of the mixtures of decalin1,3-diols

\begin{tabular}{|c|c|c|c|c|}
\hline $\begin{array}{l}\frac{\text { cis-decalin- }}{1,3-d i o l s} \\
\text { l, }\end{array}$ & $1 \mathrm{a}$ & $2 \underline{\underline{a}}$ & $\underline{\underline{a}}$ & $4 a$ \\
\hline$r_{t}(m \mid n)$ & 30,0 & 24,0 & 27,1 & 29,1 \\
\hline$\%$ & 25 & 19 & 48 & 8 \\
\hline$\frac{\text { trans-decalin- }}{1,3-d \text { tols }}$ & $\stackrel{5 a}{\leftrightarrows}$ & $\underline{6 a}$ & \multicolumn{2}{|c|}{$7 a+8 a$} \\
\hline$r_{t}(\min )$ & 21.8 & 19,9 & \multicolumn{2}{|c|}{24,6} \\
\hline$\%$ & 31 & 16 & \multicolumn{2}{|l|}{53} \\
\hline
\end{tabular}

known from the synthesis; $1 \mathrm{la}-\mathbf{4 a}$ are derivatives of cis-decalin, while $5 \mathbf{a}-8 \mathrm{a}$ are trans-decalin derivatives. This assignment facilitated the determination of the orientation of the hydroxy substituents, which could be deduced from the 'H-NMR and IR spectra. It was known from the 'H-NMR spectra of decalinols and decalin-1,4 diols, ${ }^{8}$ that the width of the signal of the $\mathrm{CH}_{2}$-groups at half-height decreases with the number of axial groups on the rings of the molecules and that the signal of the proton on the carbinol-C-atom appears at different $\delta$-values for an axial and equatorial $O H$ substituent. Similarly the stretching of the $\mathrm{C}-\mathrm{O}$ bond gives rise to absorption bands in the IR spectra with different frequencies for decalinols with axial and equatorial $\mathrm{OH}$ groups, respectively.' The band of an axial $\mathrm{OH}$ substituent appears at $960-1020 \mathrm{~cm}^{-1}$, while the same adsorption band is observed at higher frequencies for decalinols with equatorial $\mathrm{OH}$ groups. The relevant data of the 'H-NMR and IR spectra of compounds 1a-8a are presented in Table 2 .

Only one signal of the carbinol protons was observed in the ${ }^{1} \mathrm{H}-\mathrm{NMR}$ spectrum of 1a. The IR spectrum of 1a contained the $\mathrm{C}$-O-adsorption band in the lower frequency region and showed some broadening of the bands due to intramolecular $\mathrm{H}$-bonds. This identified la as cisdecalin-la,3a-diol with two axial $\mathrm{OH}$ groups. The ${ }^{1} \mathrm{H}-\mathrm{NMR}$ spectra of $3 \mathrm{a}$ and $4 \mathrm{a}$ both contained two multiplets due to the carbinol protons, and both IR spectra showed bands of the $\mathrm{C}-\mathrm{O}$-stretching vibration in the lower and upper frequency region, respectively. Consequently an axial and an equatorial $\mathrm{OH}$ substituent is present in 3a and $4 \mathbf{a}$. Therefore $\mathbf{2 a}$, which could not be isolated as a pure substance, is cis-decalin-le,3e-diol with two equatorial $\mathrm{OH}$ groups.

In the trans - decalin - 1,3 - diol series 5a and 6a gave only one multiplet signal of the protons on the carbinol-C atoms in the ${ }^{1} \mathrm{H}-\mathrm{NMR}$ spectra, and therefore contain either two axial or two equatorial $\mathrm{OH}$ groups. It was easily seen from the width of the signal of the $\mathrm{CH}_{2}$ groups in the 'H-NMR spectra and from the location of the $\mathrm{C}-\mathrm{O}$ bands in the IR spectra, that $5 \mathrm{a}$ is trans - decalin - 1a,3a - diol with two axial $\mathrm{OH}$ groups and $6 \mathrm{~g}$ is trans - decalin - 1e,3e diol with two equatorial $\mathrm{OH}$ groups, respectively. It follows that the mixture of $7 \mathbf{a}$ and $8 \mathbf{a}$ contain the remaining isomers of the trans - decalin - 1,3 - diol series with one axial and one equatorial $\mathrm{OH}$ substituent, in agreement with the location of the signals in the ${ }^{1} \mathrm{H}-\mathrm{NMR}$ and IR spectra and the chromatographic behaviour of these isomers.

While it was obvious from the spectra of $3 a$ and $4 a$ (Table 2), that these cis - decalin - 1,3 - diols each have one axial and one equatorial $\mathrm{OH}$ group, it was not possible 
Table 2. Characteristic signals in the 'H-NMR-spectra and IR-spectra of 1a-8a

\begin{tabular}{|c|c|c|c|c|}
\hline & $\begin{array}{r}{ }^{1} \mathrm{H}-\mathrm{NMR} \\
-\mathrm{CH}-\mathrm{OH} \\
(\mathrm{ppm})\end{array}$ & $\begin{array}{l}-\mathrm{CH}_{2}- \\
(\text { width, Hz) }\end{array}$ & $\begin{array}{c}\text { IR } \\
\left.-c-0-\operatorname{stretching}^{-1}\right) \\
\left(\mathrm{cm}^{-1}\right)\end{array}$ & assignment \\
\hline$\underline{\underline{1 a}}$ & 3,57 & $25 \pm 2$ & 1003,1012 & $\mathbf{a}, \mathbf{a}$ \\
\hline$\underline{\underline{3 a}}$ & 3,$67 ; 3,74$ & $20 \pm 2$ & 1014,1062 & \multirow{2}{*}{ a, } \\
\hline$\underline{\underline{a}}$ & 3,$49 ; 3,57$ & $18 \pm 2$ & 1002,1051 & \\
\hline$\underline{\underline{5 a}}$ & 3,97 & $31 \pm 2$ & 996,1016 & $\mathbf{a}, \mathbf{a}$ \\
\hline$\underline{\underline{a}}$ & 3,68 & $68 \pm 4$ & 1056,1065 & e, e \\
\hline$\underline{\underline{a}}+8 \mathrm{ga}$ & 3,$71 ; 3,95$ & $38 \pm 2$ & $998,1018,1055$ & $\mathbf{a}, \mathbf{e}$ \\
\hline
\end{tabular}

to determine definitely from the spectra the orientation of the $\mathrm{OH}$ groups at $\mathrm{C}-1$ and $\mathrm{C}-3$, respectively. Therefore $3 \mathrm{a}$ and $4 \mathrm{a}$ were transformed into decalinols of known stereochemistry by the sequence of reactions shown in Scheme 2.

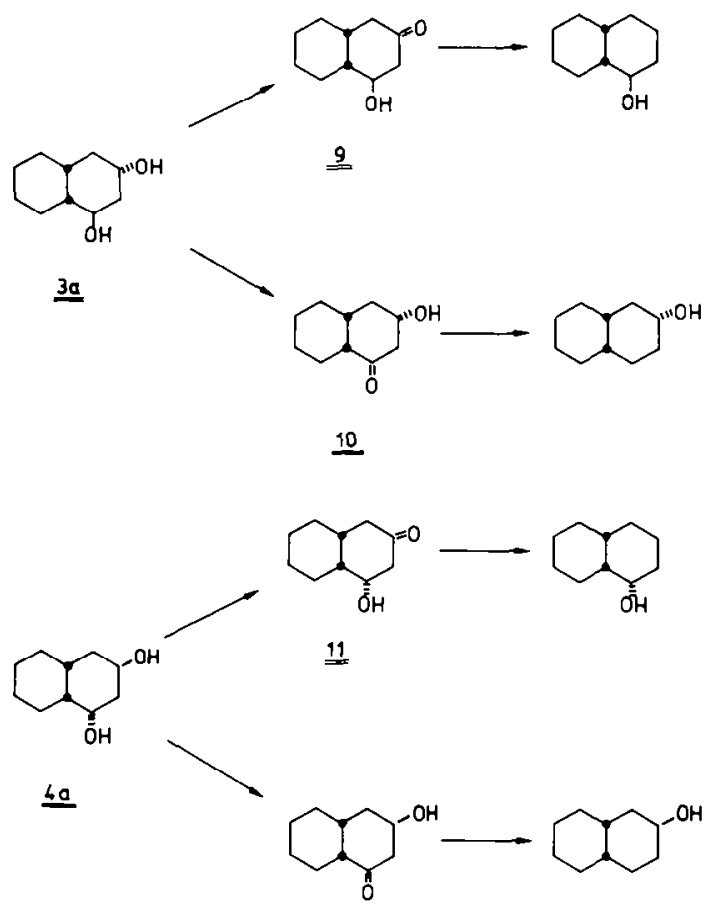

$\underline{12}$

Scheme 2.

Partial oxidation of 3a by Jones reagent yielded the isomeric ketols 9 and 10 in a ratio of about $3: 1$, which were separated by column chromatography (silica gel, benzene/acetone $6: 1, \mathrm{v} / \mathrm{v}$ ). cis - Decalin - 1a - ol was isolated after Wolff-Kishner reduction of 9 as the only decalinol, while cis - decalin - $2 \mathrm{e}$ - ol was formed from ketol 10 by the same procedure. Hence 3a is cis - decalin la,3e - diol with an axial $\mathrm{OH}$ substituent at $\mathrm{C}-1$ and an equatorial one at $\mathrm{C}-3$.

Similarly a 1:1-mixture of ketols 11 and 12 was obtained from 4a by partial oxidation with Jones reagent. The products of a Wolff-Kishner reduction of these ketols were cis - decalin - le - ol (from 11) and cis decalin - 3a - ol (from 12), respectively. $4 \mathrm{a}$ is therefore cis-decalin-le,3a-diol.
Mass spectra. In Figs. 1 and 2 the mass spectra of 1a and $5 \mathrm{a}$ are shown as typical examples. As usual ${ }^{3.10}$ the mass spectra of the stereoisomers are similar in appearance, although some variations in the ion intensities are present. However, with the exception of the intensities of the $\left[\mathrm{M}-\mathrm{H}_{2} \mathrm{O}\right]^{+}$ions, which will be discussed in the following section, it is difficult to establish that the differences in ion intensities are due to the influence of the geometry of the parent molecule on the fragmentation pathways.

The intensities of the molecular ions are small and the highest relative intensities were observed in the mass spectra of 1a and 5a. This effect could be due to a stabilisation of the molecular ions by an intramolecular H-bond" between the two axial $\mathrm{OH}$ groups of these compounds. However, a similar variation of molecular ion intensities was observed in the mass spectra of the methyl ethers 1d-8d, again 1d and $5 \mathbf{d}$ giving the greatest values. Therefore another, still unknown effect is operating besides formation of an intramolecular $\mathrm{H}$ bond. The high mass region of the spectra is dominated by the peaks of the $\left[\mathrm{M}-\mathrm{H}_{2} \mathrm{O}\right]^{+*}$ ions and $\left[\mathrm{M}-2 \mathrm{xH}_{2} \mathrm{O}\right]^{*}$ ions at $m / e$ $m / e 134$, respectively. The base peak at $m / e 67$ and most of the other peaks in the mass spectra are due to hydrocarbon ions. No complete mass shifts due to an incorporation of deuterium atoms was observed for these ions in the mass spectra of the deuterated derivatives $\mathbf{1 b}, \mathbf{c}-\mathbf{8 b}, \mathbf{c}$, therefore probably each of these ions was formed by more than one fragmentation reaction. Exceptions are the ions $m / e 70$ and $m / e$ 73. The former ions, which are of especially large relative intensities in the spectra of the trans-derivatives $5 \mathbf{a}-\mathbf{8} \mathbf{a}$, are predominantly of the elemental composition $\mathrm{C}_{4} \mathrm{H}_{6} \mathrm{O}$ and their masses are shifted to $m / e 71$ in the mass spectra of the deuterated compounds $\mathbf{1 b , c - 8 b , c . ~ C o n s e q u e n t l y ~ t h e s e ~ i o n s ~ c o n t a i n ~}$ one $\mathrm{OH}$ group and one of the $\mathrm{H}$ atoms of the carbinol group, their structure being probably that of 1 - or 2-hydroxybutadiene.

The elemental composition $\mathrm{C}_{3} \mathrm{H}_{5} \mathrm{O}_{2}$ and the complete mass shifts by 2 m.u. in the spectra of $1 \mathbf{l b , c - 8 b , c}$ show, that the ions $m / e 73$ are dihydroxy allyl ions, which arise from the molecular ions by the typical fragmentation of cyclic alcohols. ${ }^{12}$ The peak at $m / e 73$ is of diagnostic value, because ions of this type are only formed in the mass spectra of 1,3-diols. Therefore the decalin-1,3-diols can be distinguished from their positional isomers by this peak.

The mass spectrometric fragmentations are not very much altered by 0 -methylation of the decalin-1,3-diols, as was shown by the mass spectra of the 1,3-dimethoxy decalin Id and $5 d$ in Figs. 3 and 4.

Most of the intense ions in the lower mass region are 


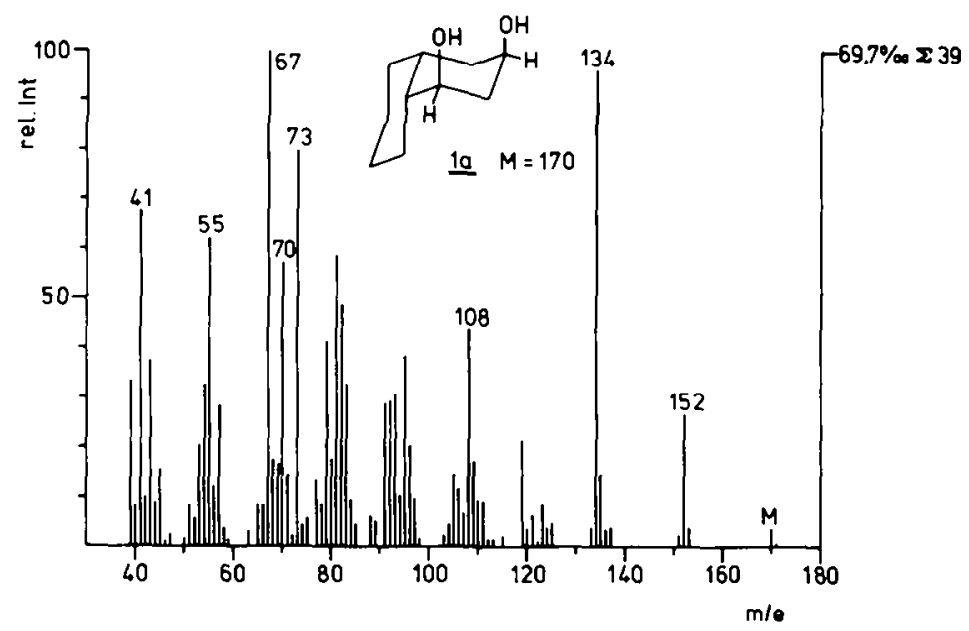

Fig. 1. $70 \mathrm{eV}$-mass spectrum of cis-decalin-1a,3a-diol 1a.

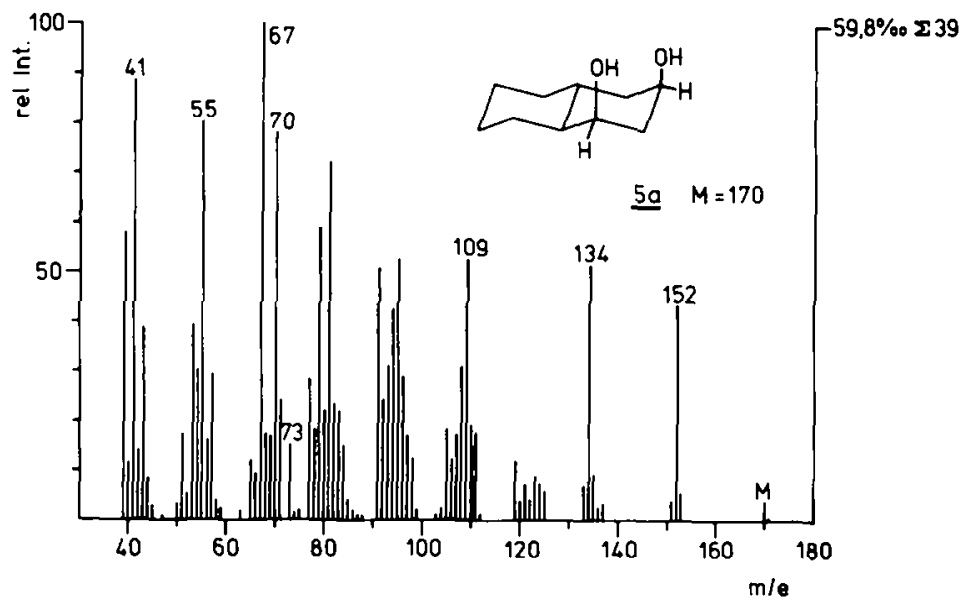

Fig. 2.70 eV-mass spectrum of trans-decalin-1a,3a-diol 5a.

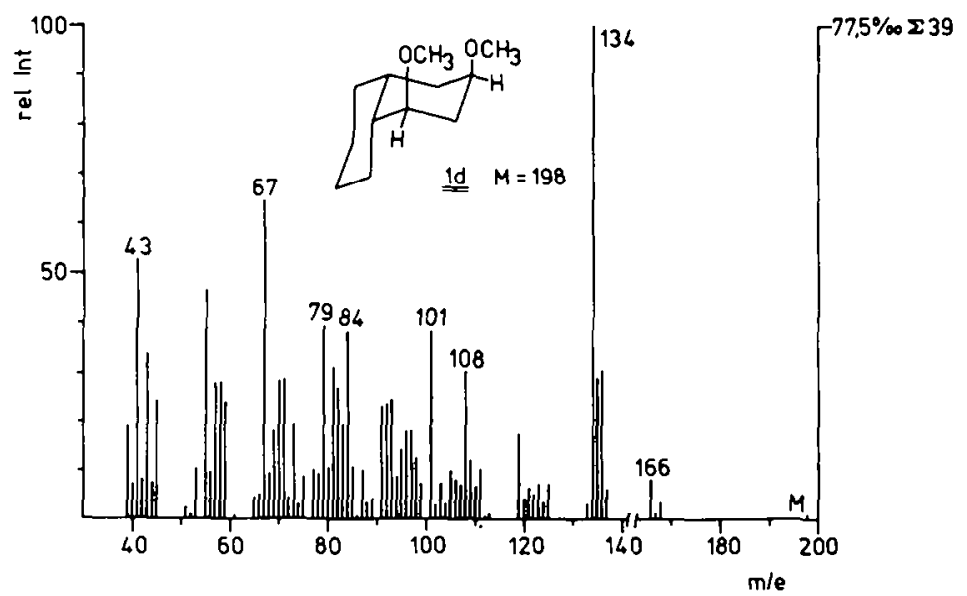

Fig. 3. 70 eV-mass spectrum of cis-1a,3a-dimethoxy decalin 1d.

again of the hydrocarbon type, exceptions are the methoxybutadiene ions $m / e 84$ and the dimethoxy allyl ions $m / e$ 101. The relative intensities of the latter ions are rather small in the mass spectra of the 1,3 - dimethoxy trans - decalins, and it may be difficult to distinguish these compounds from their positional isomers by this peak, because small peaks at $m / e 101$ are also observed in the mass spectra of 1,4-dimethoxy decalins. ${ }^{3}$ The high mass regions of the spectra contain peaks of the ions $\mathbf{M}^{+}$, [M- $\left.\mathrm{CH}_{3} \mathrm{OH}\right]^{+}$and $\left[\mathrm{M}-2 \mathrm{xCH}_{3} \mathrm{OH}\right]^{+}$, as expected, at $\mathrm{m} / \mathrm{e}$ 198,166 and 134, respectively, and in the case of the isomers $1 \mathrm{~d}, \mathbf{2 d}$ and $\mathbf{5 d}$ with a cis-orientation of the two 


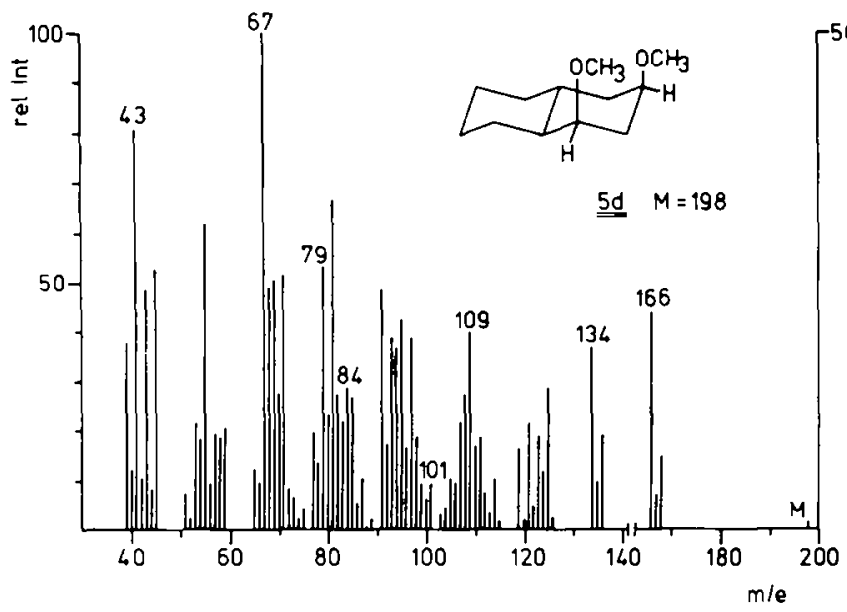

Fig. 4. $70 \mathrm{eV}$-mass spectrum of trans-1a,3a-dimethoxy decalin $5 \mathrm{~d}$.

OMe groups, additional peaks of the ions $\left[\mathrm{M}-\mathrm{CH}_{2} \mathrm{O}\right]^{+}$and $\left[\mathrm{M}-\mathrm{CH}_{2} \mathrm{O}-\mathrm{CH}_{3} \mathrm{OH}\right]$ at $m / e \quad 168$ and $m / e$ observed.

Steric effects on the elimination reactions. The relative intensities of the $\left[\mathrm{M}-\mathrm{H}_{2} \mathrm{O}\right]^{+}$ions in the mass spectra of $12-8 \mathrm{a}$ and the distribution of the ion current of these ions among the species $\left[\mathrm{M}-\mathrm{H}_{2} \mathrm{O}\right]^{+},[\mathrm{M}-\mathrm{HDO}]^{+}$and $\left[\mathrm{M}-\mathrm{D}_{2} \mathrm{O}\right]^{+}$ in the mass spectra of $\mathbf{1 b}, \mathbf{c}-\mathbf{8 b}, \mathbf{c}$ is shown in Table 3 .

From the various fragmentations of intact molecular ions of cyclic diols forming $\left[\mathrm{M}-\mathrm{H}_{2} \mathrm{O}\right]^{+}$ions two reactions are distinguished by specific steric requirements of the transition states. The first one involves an $\mathrm{OH}$ group and the $\mathrm{H}$ atom bound to the $\mathrm{C}$ atom of the other carbinol group. As the dissociation energy of this $\mathrm{C}-\mathrm{H}$ bond is about $20 \mathrm{kcal} / \mathrm{mole}$ lower than that of the $\mathrm{C}-\mathrm{H}$ bonds of $\mathrm{CH}_{2}$ groups in alkanes or cycloalkanes, ${ }^{13}$ this $\mathrm{H}_{2} \mathrm{O}$ elimination has the lowest activation energy. However, as $\mathrm{OH}$ group and $\mathrm{H}$ atom have to approach each other rather closely in the transition state, the $\mathrm{H}_{2} \mathrm{O}$ elimination by this mechanism requires a trans-orientation of the two $\mathrm{OH}$ groups in cyclic 1,3-diols ${ }^{2}$ (Scheme 3).

This steric condition is met by the decalin - 1,3 - diols $3 \mathbf{a}, 4 \mathbf{a}, 7 \mathbf{a}$ and $8 \mathbf{a}$, each possessing one axial and one equatorial $\mathrm{OH}$ group. As there is an additional and energetically favoured reaction path for the $\mathrm{H}_{2} \mathrm{O}$ elimina-

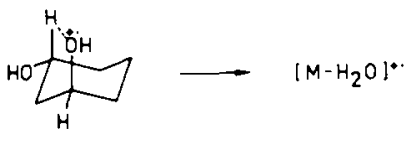

Scheme 3

tion, compared to the other stereoisomers, one expects a larger intensity of the $\left[\mathrm{M}-\mathrm{H}_{2} \mathrm{O}\right]^{+}$ions in the mass spectra of $3 \mathbf{a}, 4 \mathbf{a}, 7 \mathbf{a}$ and $8 \mathrm{a}$. Indeed, this effect was observed, although the effect is not very large in the case of the trans - decalin-1,3-diols and is not as clear as in the mass spectra of stereoisomeric decalin - 1,4 - diols. ${ }^{3}$

However, the different mechanisms of the $\mathrm{H}_{2} \mathrm{O}$ eliminations from the molecular ions are easily seen from the ratio of $\left[\mathrm{M}-\mathrm{H}_{2} \mathrm{O}\right]^{+}$ions and $[\mathrm{M}-\mathrm{HDO}]^{+}$ions in the mass spectra of the deuterated derivatives 1c-8c (Table 3 ). About $80-83 \%$ of the $\mathrm{H}_{2} \mathrm{O}$ molecules are lost by a 1,3-elimination from the cis - decalin - 1,3-diols $3 \mathbf{a}$ and $\mathbf{4 a}$, as is shown by the corresponding values of the loss of HDO from $3 c$ and $4 c$, while less than 5\% loss of HDO is observed in the mass spectra of $1 c$ and $2 c$. A 1,3elimination of $\mathrm{HDO}$ is not possible from the intact molecular ions of these isomers, but has to be preceded by cleavage of the hydroxylated ring. If in the mass spectra

Table 3. Relative ion intensities and deuterium retention of eliminations of water from molecular ions of decalin-1,3-diols (corrected for ${ }^{13} \mathrm{C}$ )

\begin{tabular}{|c|c|c|c|c|c|c|c|c|}
\hline & & $\underline{\underline{1 a}}$ & $2 \mathrm{a}$ & $3 a$ & $4 \mathrm{a}$ & $\underline{\underline{5 a}}$ & $\underline{\underline{6 a}}$ & $\underline{\underline{7 a}}+\underline{\underline{8 a}}$ \\
\hline \multirow{3}{*}[\mathrm{M}-\mathrm{H}_{2}\mathrm{O}]{$^{+\cdot}$} & $\%$ B $* 1$ & 26,7 & 37.7 & 66.7 & 63,8 & 43,7 & 53,8 & 63,6 \\
\hline & $\overline{\phi(T * *)}$ & 18,6 & 22,3 & 44,2 & 47,6 & 26,2 & 32,0 & 36,2 \\
\hline & & $\underline{1 b}$ & $\underline{2 b}$ & $3 \mathrm{~b}$ & 40 & $\underline{5 \mathrm{~b}}$ & $6 \mathrm{~b}$ & $7 b+8 b$ \\
\hline$\left[M-D_{2} O\right]^{+}$ & & $90 \%$ & $20 \%$ & - & - & $90 \%$ & - & $6 \%$ \\
\hline \multirow{2}{*}{\multicolumn{2}{|c|}{$[M-\mathbf{M} D 0]^{+\cdot}$}} & $10 \%$ & $80 \%$ & $>95 \%$ & $>95 \%$ & $10 \%$ & $>95 \%$ & $94 \%$ \\
\hline & & $\underline{\underline{1 c}}$ & $2 c$ & $\underline{3 c}$ & $\underline{\underline{4 c}}$ & $\underline{\underline{5 c}}$ & $\underline{\underline{6 c}}$ & $7 c+8 c$ \\
\hline$[\mathrm{M}-\mathrm{BDO}]^{+}$ & & - & - & $83 \%$ & $81 \%$ & $9 \%$ & - & $71 \%$ \\
\hline$\left[\mathrm{M}-\mathrm{H}_{2} \mathrm{O}\right]^{+}$ & & $>95 \%$ & $>95 \%$ & $17 \%$ & $19 \%$ & $91 \%$ & $>95 \%$ & $29 \%$ \\
\hline
\end{tabular}

-) \% base peak

*) \% total ion current 
of $3 c$ and $4 c$ the 1,3-elimination occurs to a similar percentage from ring opened molecular ions, at least $75 \%$ of the $\mathrm{H}_{2} \mathrm{O}$ molecules are eliminated by a stereospecific process from the molecular ions of $\mathbf{3 a}$ and $\mathbf{4 a}$. Similar results have been obtained from the mass spectra of decalin - 1,4 - diols. ${ }^{3}$

The steric control of the $\mathrm{H}_{2} \mathrm{O}$ elimination is somewhat less in the mass spectra of the trans - decalin - 1,3 - diols $7 a$ and $8 \mathrm{a}$. The data of Table 3 indicate, that about $70 \%$ of the $\mathrm{H}_{2} \mathrm{O}$ molecules are lost by a 1,3-elimination from the mixture of molecular ions of 7a and $8 \mathbf{a}$, and more than $60 \%$ are lost by a stereospecific elimination from intact molecular ions. This slight reduction in the stereospecifity is probably due to the more rigid skeleton of the trans decalin - 1,3 - diols.

The second specific mechanism of the $\mathrm{H}_{2} \mathrm{O}$ elimination from molecular ions of cyclic diols corresponds to the formation of the $\mathrm{H}_{2} \mathrm{O}$ molecules by interaction between the two OH groups (Scheme 4). In the case of intact

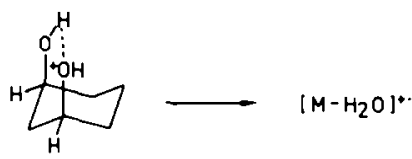

Scheme 4.

molecular ions of cyclic 1,3-diols, the short distance, which is a prerequisite for the elimination of $\mathrm{H}_{2} \mathrm{O}$ by this mechanism, is only possible in isomers with a diaxial orientation of the 1,3-OH substituents. The occurence of this mechanism is easily established by elimination of $\mathrm{D}_{2} \mathrm{O}$ in the mass spectra of the $\mathrm{O}-\mathrm{d}_{2}$ derivatives of diols.

The ground state conformations of the cis - decalin - 1,3diol 1a and the trans - decalin - 1,3 - diol 5a are convenient for this reaction. In the case of the cis decalin - 1,3 - diol 2a the required conformation of the transition state can be achieved by a chair-chair interconversion of the cis-decalin system. However, the $\Delta \mathrm{H}_{\mathrm{f}}$ of this conformation is certainly larger by some $\mathrm{kcal} / \mathrm{mols}$ than that of the ground state conformation, because of severe 1,3-diaxal repulsions between the $\mathrm{OH}$ substituents and the fused second ring. ${ }^{14} \mathrm{~A}$ rather short distance between the $\mathrm{OH}$ groups of the trans - decalin 1,3 - diol $6 \mathrm{a}$ is possible, if the hydroxylated ring adopts a boat conformation. But again this conformation of the molecular ion is energetically not favoured. In the case of the remaining isomers $3 \mathbf{a}, \mathbf{4 a}, 7 \mathbf{a}$ and $\mathbf{8 a}$, no conformation of the molecular ions has a sufficiently short distance between the $\mathrm{OH}$ groups.

The different steric situation in the stereoisomeric decalin - 1,3 - diols is nicely demonstrated by the amount of $\mathrm{D}_{2} \mathrm{O}$ lost from the molecular ions of the $\mathrm{O}-\mathrm{d}_{2}$ derivatives $1 \mathrm{~b}-8 \mathrm{~b}$ (Table 3 ). No loss of $\mathrm{D}_{2} \mathrm{O}$ was observed within the limits of error in the mass spectra of $3 \mathrm{~b}$ and $\mathbf{4 b}$ and only about $6 \%$ in the mass spectrum of the mixture of $\mathbf{7 b}+\mathbf{8 b}$. In contrast to this the peak $\left[\mathrm{M}-\mathrm{D}_{2} \mathrm{O}\right]^{+}$dominates in the spectra of $1 \mathbf{b}$ and $5 \mathbf{b}$, the percentages of $\mathrm{D}_{2} \mathrm{O}$ elimination are $\mathrm{ca} 90 \%$. A similar participation of the interaction of two $\mathrm{OH}$ groups in the elimination of $\mathrm{H}_{2} \mathrm{O}$

†It has been suggested by a referee, that in this case the ratio of [M-HDO] ${ }^{\prime} /\left[\mathrm{M}-\mathrm{D}_{2} \mathrm{O}\right]^{\prime}{ }^{\prime}$ in the mass spectrum of $2 \mathrm{~b}$ should vary by reducing the energy of the jonizing electrons. Within the limits of error no such variations have been observed between 70 and $17 \mathrm{eV}$. Probably the energy distribution of the molecular ions is not sufficiently altered by this method to observe any effect on these processes with a low activation energy. was observed in the mass spectra of decalin - 1,4- diols of appropriate geometry. ${ }^{3}$ An interaction between the $\mathrm{OH}$ groups in these 1,4-diols is only posible, if the hydroxylated ring has a boat conformation. Obviously the necessity to change the conformation of the molecular ions prior to the $\mathrm{H}_{2} \mathrm{O}$ elimination has no large effect on the course of the reaction in the case of the decalin - 1,4 diols. A corresponding change of the conformation of the hydroxylated ring has to take place in the molecular ions of the trans - decalin - 1,3 - diol 6b prior to elimination of $\mathrm{D}_{2} \mathrm{O}$, although the distance between the two OD-groups is still rather large. No elimination of $\mathrm{D}_{2} \mathrm{O}$ was detected within the limits of error in the mass spectrum of $\mathbf{6 b}$, so a transition state with a suitable geometry for the interaction between the $\mathrm{OH}$ groups is not very likely for the molecular ions of $\mathbf{6 a}$.

The cis - decalin - 1,3 - diol $2 \mathrm{a}$ is the other isomer, which has to change its ground state conformation to permit interaction between the two $\mathrm{OH}$ groups. In the mass spectrum of the $\mathrm{O}-\mathrm{d}_{2}$ derivative $2 \mathrm{~b}$ about $20 \%$ elimination of $\mathrm{D}_{2} \mathrm{O}$ was observed besides loss of HDO. Hence in contrast to the results obtained from the mass spectra of decalin - 1,4 - diols the amount of $\mathrm{H}_{2} \mathrm{O}$ lost from the molecular ions of decalin - 1,3 - diols by interaction of both $\mathrm{OH}$ groups is considerably reduced if a change of the conformation of the molecular ions is a prerequisite of this reaction.

The $20 \%$ loss of $\mathrm{D}_{2} \mathrm{O}$ from molecular ions of $2 \mathrm{~b}$ can be explained in two ways. Firstly an equilibrium between the conformations $\mathbf{2 b}$ and $\mathbf{2} \mathbf{b}^{\prime}$ prior to ionisation may be assumed in the gas phase of the inlet system and ions source of the mass spectrometer, and after ionisation only 2b' loses $\mathrm{D}_{2} \mathrm{O}$.

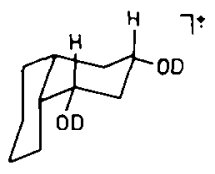

$\underline{2 b}$

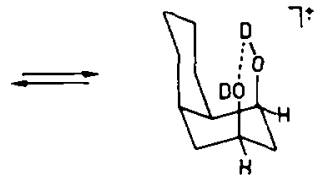

$\underline{2 b^{*}}$
Scheme 5 .

In this case the ratio 4 of $[\mathrm{M}-\mathrm{HDO}]^{+} /\left[\mathrm{M}-\mathrm{D}_{2} \mathrm{O}\right]^{+*}$ should reflect the composition of the mixture at the equilibrium before ionisation, i.e. $80 \% \mathbf{2 b}$ and $20 \% 2 \mathrm{~b}^{\prime}$. The difference in energy between $\mathbf{2 b}$ and $\mathbf{2} \mathbf{b}^{\prime}$ is not known, but has to be less than $1,5 \mathrm{kcal} / \mathrm{Mol}$ to account for the presence of $20 \%$ $2 \mathbf{b}^{\prime}$. With respect to the steric repulsion between the axial substituents in $\mathbf{2} \mathbf{b}^{\prime}$ this is rather unlikely.

In the second explanation it is assumed, that nearly all molecules are in the conformation $\mathbf{2 b}$ before ionisation, but that conformational changes take place in the molecular ions after ionisation. The transition state of $\mathrm{D}_{2} \mathrm{O}$ elimination is not necessarily conformation $\mathbf{2} \mathbf{b}^{\prime}$, because in the flexible cis-decalin system a short distance between both $\mathrm{OH}$ groups is already possible in a boat conformation. The excess energy implanted into the molecular ions during ionisation by $70 \mathrm{eV}$ electrons is certainly large enough to activate these conformational changes, however changing the conformation has to compete with other fragmentation reactions with low activation energies. This accounts for the reduction in the amount of stereospecific loss of $\mathrm{D}_{2} \mathrm{O}$ from $\mathbf{2 b}$, compared with the same reaction of $\mathbf{1 b}$. This explanation is in accord with stereospecific reactions in the mass spectra of stereoisomeric 4-t.-butyl- and 4 - methyl - cyclohexane 1,3 - diols. ${ }^{4.5} \dagger$ 
In the mass spectra of di - $\mathrm{O}$ - methyl ethers of cyclic diols, two elimination reactions were also observed which are controlled by the geometry of the molecular ions. The first one is a methanol elimination with a low activation energy, which involves an OMe group and the $\mathrm{H}$ atom of the other $\mathrm{CH}$-carbinol group; analogous to Scheme 3, and which can take place in the 1,3-dimethoxy - decalins 3d, 4d, 7d and 8d. For similar reasons as with the corresponding 1,3-diols one expects an enhanced intensity of the $\left[\mathrm{M}-\mathrm{CH}_{3} \mathrm{OH}\right]^{+}$ions in the mass spectra of $3 \mathrm{~d}, \mathbf{4 d}, \mathbf{7 d}$ and $8 d$. However, as is seen by the data of Table 4, this enhancement is rather small.

Nevertheless the mass spectra of the deuterated derivatives 1e-8e show the expected differences in the mechanism of the methanol eliminations. The molecular ions of $3 \mathrm{~d}$ and $\mathbf{4 d}$ lose $76 \% \mathrm{MeOD}$ and $72 \% \mathrm{MeOD}$, respectively, and those of $7 \mathbf{d}+\mathbf{8 d}$ eliminate $65 \% \mathrm{MeOD}$, while only about $10 \%$ loss of MeOD was observed from the molecular ions of the other isomers, where no interaction between a $\mathrm{MeOH}$ group and a carbinol- $\mathrm{CH}$ group is possible in the intact molecular ions. Calculations based on these data show that about $70 \%, 63 \%$ and $57 \%$ of the elimination of methanol from $3 \mathbf{d}, \mathbf{4 d}$ and $7 \mathbf{d}+\mathbf{8 d}$, respectively, occurs by a stereospecific process in intact molecular ions, in agreement with the extent of stereospecifity of this reaction in the mass spectra of 1,4 . dimethoxy - decalins ${ }^{3}$ and dimethoxy cyclohexanes. ${ }^{2}$

The second reaction in the mass spectra of dimethoxycycloalkanes for which the steric control of the mechanism is known ${ }^{2}$ corresponds to a loss of $\mathrm{CH}_{2} \mathrm{O}$ from the molecular ions. The crucial step of this reaction is the transfer of a $\mathrm{H}$ atom from one $\mathrm{MeO}$ group to the second one. The short distance required by this transfer is only possible in a 1,3-dimethoxycycloalkane with both $\mathrm{MeO}$ substituents in an axial position (Scheme 6).

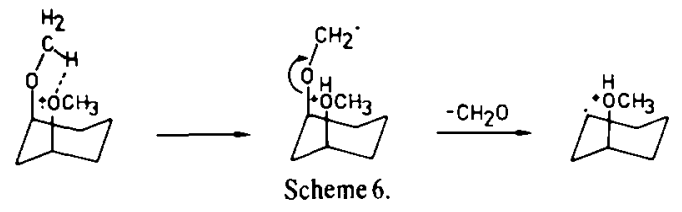

In accord with this mechanism distinct peaks of $\left[\mathrm{M}-\mathrm{CH}_{2} \mathrm{O}\right]^{+}$ions at $m / e 168$ were observed in the mass spectra of 1d and 5d, and were completely absent in the spectra of $3 d, 4 d$ and $7 d+8 d$ (Figs. 3 and 4 and Table 4). A small ion current persists at $m / e 168$ in the mass spectra of $2 \mathrm{~d}$ and $6 \mathrm{~d}$ after corrections for the contribution of ${ }^{13} \mathrm{C}$ containing ions to this $m / e$-value, but the corrected relative intensities of $1.6 \%$ and $2.3 \%$, respectively, are too small to prove the formation of $\left[\mathrm{M}-\mathrm{CH}_{2} \mathrm{O}\right]^{+}$ions. If at all, a small amount of these ions may be formed in the mass spectrum of $2 \mathrm{~d}$. Again the necessity of the molecular ions to change their conformation before the fragmentation reduces the probability of this fragmentation pathway drastically.

It is of interest to note that the intensity variation of the $\left[\mathrm{M}-2 \mathrm{xH}_{2} \mathrm{O}\right]^{+}$and $\left[\mathrm{M}-2 \mathrm{xCH}_{3} \mathrm{OH}\right]^{+}$-ions in the mass spectra of 19-8a and 1d-8d is quite different from that of the $\left[\mathrm{M}-\mathrm{H}_{2} \mathrm{O}\right]^{+}$- and $\left[\mathrm{M}-\mathrm{CH}_{3} \mathrm{OH}\right]^{+\cdot}$-ions (Table 5).

Table 4. Relative ion intensities and deuterium retention of eliminations from molecular ions of 1,3-dimethoxy decalins (corrected for ${ }^{13} \mathrm{C}$ )

\begin{tabular}{|c|c|c|c|c|c|c|c|c|}
\hline & & $\underline{\underline{1 d}}$ & $\underline{\underline{2 d}}$ & $3 \mathrm{~d}$ & $\underline{\underline{d d}}$ & $\underline{\underline{5 d}}$ & $\underline{\underline{6 d}}$ & $\underline{7 d}+8 d$ \\
\hline \multirow{2}{*}[\mathrm{M}-\mathrm{CH}_{3}\mathrm{OH}]{$^{+\cdot}$} & $\left.\% \mathrm{~B}^{*}\right)$ & 8,4 & 11,6 & 16,6 & 16,2 & 43,8 & 42,1 & 68,4 \\
\hline & \%ot**) & 6,5 & 9,2 & 12,2 & 12,4 & 24,7 & 23,4 & 38,7 \\
\hline \multirow{2}{*}[\mathrm{M}-\mathrm{CH}_{2}\mathrm{O}]{$^{+.}$} & $\left.\% \mathrm{~B}^{*}\right)$ & 4,0 & $(1,6)$ & - & - & 14,9 & $(2,3)$ & - \\
\hline & $\left.\% T^{*} *\right)$ & 3,1 & $(1,2)$ & - & - & 8,4 & $(1,2)$ & - \\
\hline & & $\underline{\underline{1}}$ & $\stackrel{2 e}{=}$ & $\underline{\underline{3 e}}$ & 4e & $\underline{\underline{5 e}}$ & $\underline{\underline{60}}$ & $\underline{\underline{7 e}}+8 \theta$ \\
\hline$\left[\mathrm{M}-\mathrm{CH}_{3}, \mathrm{OD}\right]^{+}$ & & $12 \%$ & $8 \%$ & $76 \%$ & $72 \%$ & $13 \%$ & $12 \%$ & $65 \%$ \\
\hline$[\mathrm{M}-\mathrm{CH}, \mathrm{OH}]^{+}$ & & $88 \%$ & $92 \%$ & $24 \%$ & $28 \%$ & $87 \%$ & $88 \%$ & $35 \%$ \\
\hline
\end{tabular}

*) \% baso poak

**) \% total ton current

Table 5. Relative intensities of $\left[\mathrm{M}-2 \mathrm{xH}_{2} \mathrm{O}\right]^{+}$and $\left[\mathrm{M}-2 \mathrm{xCH}_{3} \mathrm{OH}\right]^{+}$ions

\begin{tabular}{|c|c|c|c|c|c|c|c|c|}
\hline & & $\underline{\underline{1 a}}$ & $\underline{\underline{2 a}}$ & $\underline{\underline{3 a}}$ & $\underline{\underline{4 a}}$ & $\underline{\underline{5 a}}$ & $\underline{\underline{a}}$ & $\underline{7 a}+8 a$ \\
\hline \multirow{2}{*}[M-2\times\mathrm{H}_{2}\mathrm{O}]{$^{+-}$} & \%B*) & 96,0 & 44,6 & 24,9 & 32,2 & 51,8 & 59,0 & 60,2 \\
\hline & $\left.\phi T^{* *}\right)$ & 66,9 & 29,5 & 16,5 & 24,0 & 31,0 & 35,0 & 34,2 \\
\hline & & $\underline{\underline{l d}}$ & $2 \mathrm{~d}$ & $\underline{\underline{3 d}}$ & $4 d$ & sd & 6d & $7 d+8 d$ \\
\hline \multirow{2}{*}[\mathrm{M}-2\times\mathrm{CH}_{3}\mathrm{OH}]{$^{+.}$} & $\left.\% \mathrm{~B}^{*}\right)$ & 100,0 & 60,9 & 44,3 & 49,8 & 36,9 & 38,8 & 36,7 \\
\hline & $\not \gamma * *)$ & 77,5 & 48,1 & 32,8 & 38,9 & 20,9 & 21,6 & 20,8 \\
\hline
\end{tabular}

*) X base peak

**) \# total ion current 
Large intensities of $\left[\mathrm{M}-2 \mathrm{xH}_{2} \mathrm{O}\right]-$ or $\left[\mathrm{M}-2 \mathrm{xCH}_{3} \mathrm{OH}\right]^{+}$ ions were found in the mass spectra of isomers with relatively small intensities of the primary fragment ions of $\left[\mathrm{M}-\mathrm{H}_{2} \mathrm{O}\right]^{+}$or $\left[\mathrm{M}-\mathrm{CH}_{3} \mathrm{OH}\right]^{+*}$ and vice versa. This indicates, that many of the primary fragment ions decompose rapidly by loss of a second $\mathrm{H}_{2} \mathrm{O}$ or $\mathrm{MeOH}$ molecule. However, most of the $\left[\mathrm{M}-2 \mathrm{xH}_{2} \mathrm{O}\right]^{+}$and $\left[\mathrm{M}-2 \mathrm{xCH}_{3} \mathrm{OH}\right]^{+}$ ions in the mass spectra of $1 \mathrm{c}-8 \mathrm{c}$ and le-8e, respectively, retain both $\mathrm{D}$ atoms and consequently are formed by sterically unspecific processes. This result is in keeping with the Quasi-Equilibrium-Theory of mass spectrometry, because the sterically controlled eliminations from the molecular ions of cyclic diol derivatives are reactions with low activation energies. Hence molecular ions with a large excess energy react rapidly with a low selectivity and the excess energy is large enough for a further decomposition of the primary fragment ions, while molecular ions with a small excess energy form with a higher selectivity stable fragment ions by low energy processes.

\section{CONCLLSION}

The investigation of the mass spectrometric fragmentations of the stereoisomeric decalin - 1,3 - diols 1a-8a and 1,3-dimethoxydecalins 1d-8d with the aid of deuterated derivatives demonstrates, that the molecular ions of these compounds decompose by stereospecific elimination reactions similar to the fragmentation of molecular ions of cyclohexane - 1,3- diol and its derivatives. Obviously the extent of stereospecific elimination is not influenced very much by the attachment of a second saturated ring, if the ground state conformation of the decalin - 1,3 - diol corresponds to the spatial arrangement of the transition state of the elimination reaction. However, contrary to cyclohexanediols and decalin - 1,4-diols or their O-Me derivatives, the amount of ions formed by stereospecific eliminations is reduced drastically if conformational changes of the molecular ions are required before the fragmentation. The difference in the ion intensities and in the incorporation of $\mathrm{D}$ labels into the ions formed by elimination of water or methanol can be used to distinguish between the stereoisomers of decalin - 1,3 diol and 1,3 - dimethoxy decalin by mass spectrometry.

\section{EXPERIMENTAL}

All m.ps are uncorrected. IR spectra were taken in $\mathrm{KBr}$ pellets with a Perkin-Elmer 137 spectrophotometer. NMR spectra were measured on a $60 \mathrm{MHz}$. NMR spectrometer Varian $\mathrm{T} 60$ in $\left(\mathrm{CD}_{3}\right)_{2} \mathrm{SO}$-soln using TMS as internal reference. Gas chromatograms were obtained with a Perkin-Elmer F6 and Varian MAT 111 gas chromatograph using $50 \mathrm{~m}$ QF 1 capillary columns $\left(130^{\circ}\right.$, $1 \mathrm{ml} / \mathrm{sec} \mathrm{He}$ ).

Mass spectra were measured on a Varian MAT CH4 mass spectrometer at an electron energy of $70 \mathrm{eV}$, electron trap current $40 \mu \mathrm{A}$, ion source temp. $c a$. $200^{\circ}$. The decalin - 1,3-diols were introduced into the ion source by a vacuum lock and direct insertion probe, the 1,3-dimethoxydecalins were measured by a heated inlet system $\left(150^{\circ}\right)$. The exact masses of selected ions was determined with a Varian MAT SM $1 B$ mass spectrometer by the peak matching technique using PFK as reference. Experimental and calculated values of the ion masses agreed within $3 \mathrm{ppm}$ to the assumed elemental composition of the ions.

cis - Decalin - 1,3 - diols 1a-4a. cis - Decalin - 1,3 - dion ${ }^{6}(18.7 \mathrm{~g})$ in THF was reduced with $10 \%$ excess LAH. The crude product consisted of an octalinol as a main product and of cis - decalin 1,3 - diols (ca. 40\%). The crude mixture was used without any purification for column chromatography on silica gel and elution with acetone/benzene $=2 / 3(\mathrm{~V} / \mathrm{V})$. The fractions containing the octalinol were discarded. The remaining fractions yielded 1a $(1.72 \mathrm{~g})$ m.p. $182^{\circ}$, and $4 \mathrm{a}(0.50 \mathrm{~g})$ m.p. $147^{\circ}$, after recrystallization from benzene, and were obtained together with $4.13 \mathrm{~g}$ of a mixture of $2 \mathrm{a}$ and $3 \mathrm{a}$. By recrystallization of this mixture from benzene $3 \mathrm{a}$ $(1.71 \mathrm{~g})$, m.p. $168^{\circ}$, was obtained. The combined benzene solns from the recrystallization of $3 \mathrm{a}$ were evaporated to give about $2 \mathrm{~g}$ of a mixture of $3 \mathrm{a}$ and $2 \mathrm{a}(52 \%: 48 \%)$.

trans - Decalin - 1,3 - diols 5a-8n. LAH-reduction (10\% excess) of trans - decalin - $1,3-$ dion $^{6}(21.4 \mathrm{~g})$ in THF gave a crude product, from which a mixture of $5 \mathrm{~g}-8 \mathrm{a}(16.7 \mathrm{~g})$ was obtained by column chromatography on silica gel and elution with acetone/benzene $=2 / 3(\mathrm{~V} / \mathrm{V})$. The bis-TMS ether of the diols were obtained from the mixture by reaction with $\mathrm{N}$ - bis - TMS acetamide, dilution with $\mathrm{H}_{2} \mathrm{O}$ and extraction with $\mathrm{n}$-hexane. ${ }^{15}$ The resulting mixture was separated by column chromatography on silicagel and elution with acetone/benzene $=1 / 3(\mathrm{~V} / \mathrm{V})$ into three fractions. Each fraction was purified by column chromatography using the same conditions as before. After evaporation of the solvent the residual oil of each fraction was redissolved in water/MeOH 1/1 (V/V) and heated under reflux for $1 \mathrm{hr}$. By evaporation to dryness and recrystallization from EtOH $\mathbf{5 a}$ $(2.63 \mathrm{~g})$ m.p. $189^{\circ} ; 6 \mathrm{a}(1.45 \mathrm{~g}) \mathrm{m} . \mathrm{p} .155^{\circ}$; and a mixture of $7 \mathrm{a}$ and $8 \mathrm{a}$ $(4.61 \mathrm{~g})$ were obtained.

1,3-Dimethoxy - decalins 1d-8d. The individual isomers of the diols $1 a-6 a$ and the mixtures of $2 a+3 a$ and $7 a+8 a$, respectively, were dissolved in $\mathrm{CH}_{2} \mathrm{Cl}_{2}(30 \mathrm{ml} / \mathrm{g}$ diol) and methylated by the $\mathrm{CH}_{2} \mathrm{~N}_{2} / \mathrm{BF}_{3} / \mathrm{ether}$ complex. ${ }^{16}$ After the usual work up of the mixture the 1,3-dimethoxydecalins were purified by vacuum destillation.

Decalin - 1,3 - diol - bis - trifluoracetates. For gas chromatographic analysis the mixtures of the diols were transformed into the corresponding trifluoracetates by dissolving in trifluor-acetic acid anhydride (ca. 10 times in excess). After $5 \mathrm{~min}$ most of the anhydride and of trifluor-acetic acid was removed by vacuum destillation and the residues used for gas chromatog. raphy.

Deuterated derivatives. The decalin - 1,3- diols $-1,3-d_{2}$ Ic-8c were synthesized by the same methods as 1a-8a, using $L A D$ for the reduction.

Deuterium content (hy mass spectrometry)

$\begin{array}{llllllll}\text { lc } & 99 \% & d_{2} ; & 1 \% & d_{1} ; & 5 c & 96 \% d_{2} ; & 4 \% d_{1} ; \\ 2 c+3 c & 98.5 \% & d_{2} ; & 1.5 \% d_{1} ; & 6 c & 98 \% d_{2} ; & 2 \% d_{1} ; \\ 3 c & 99 \% & d_{2} ; & 1 \% & d_{1} ; & 7 c+8 c & 98 \% d_{2} ; & 2 \% d_{1} ; \\ 4 c & 99 \% & d_{2} ; & 1 \% & d_{1} ; & & & \end{array}$

The 1,3 - diemthoxydecalins - 1,3- $d_{2}$ le-se were obtained from the correspondingly deuterated diols by $\mathrm{O}$-methylation as described before. The decalin - 1,3-diol - $0-d_{2}$ derivatives $1 \mathbf{b}-\mathbf{8 b}$ were obtained by dissolving $12-8 \mathrm{a}$, respectively, in a small amount of THF and adding $\mathrm{D}_{2} \mathrm{O}$ ( $99.9 \%$ deuterium) in a very large excess. After mixing for $1 \mathrm{hr}$ the solns were evaporated to dryness and the residues introduced into the mass spectrometer after preconditioning of the instrument with $\mathrm{D}_{2} \mathrm{O}$. Deuterium content (by mass spectrometry) $95 \% \mathrm{~d}_{2} ; 5 \% \mathrm{~d}_{1}$.

Partial oxidation of 3a. 3a $(856 \mathrm{mg})$ in THF was oxidized by Jones reagent analogous to the method of Bec et al. ${ }^{17} \mathrm{~A}$ mixture of $360 \mathrm{mg}(-42 \%)$ of ketols $9+10$ was obtained, which was separated into $276 \mathrm{mg} 9$ and $82 \mathrm{mg} 10$ by column chromatography on silica gel and elution with acetone/benzene $=1 / 6(\mathrm{~V} / \mathrm{V}) ; 9$ m.p. $54^{\circ} ; 10$ m.p. $18^{\circ}$ after recrystallization from benzene.

Wolf-Kishner-reduction of 9. From the Wolff-Kishnerreduction of $9(276 \mathrm{mg})$ by standard procedures, ${ }^{18} 77 \mathrm{mg}(28 \%)$ of decalin - la - ol were obtained after purification by column chromatography (silica gel, acetone/benzene $=1 / 2(\mathrm{~V} / \mathrm{V})$ ) and identified by comparison of its IR spectrum with that of an authentic sample.

Wolf-Kishner-reduction of 10 . Decalin - $2 \mathrm{e}-$ ol $25 \mathrm{mg}$ (31\%) was obtained from $10(82 \mathrm{mg})$ and identified by the same methods.

Partial oxidation of $\mathbf{4 a}$. By the methods mentioned for the oxidation of 3a a mixture of ketols $11+12(132 \mathrm{mg} ; 49 \%)$ was obtained from $4 \mathrm{a}(269 \mathrm{mg})$, which was separated into $11(68 \mathrm{mg})$, m.p. $93^{\circ}$ and $12(63 \mathrm{mg})$, m.p. $103^{\circ}$ after recrystallization from benzene.

Wolf-Kishner-reduction of 11. As described above the reduction of $11(68 \mathrm{mg})$ gave decalin-le-ol $(30 \mathrm{mg} ; 45 \%$; after purification), which was identified by its IR spectrum. 
Wolf-Kishner-reduction of 12. After reduction and purification, as described before, decalin-2a-ol ( $23 \mathrm{mg} ; 36 \%$ ) was obtained from $12(63 \mathrm{mg})$ and identified by its IR spectrum.

Acknowledgement-This work was supported by Zentralmitteln des Ministers für Wissenschaft und Forschung des Landes Nordrhein-Westfalen.

\section{RETERENCES}

'H.-F. Grützmacher, Suom. Kemi. 46. 50 (1973).

${ }^{2}$ H.-F. Grützmacher and J. Winkler, Org. Mass Spectrom. 3, 1139 (1970).

${ }^{3}$ H.-F. Grützmacher and K.-H. Fechner, Ibid. 7, 573 (1973)

${ }^{4}$ H.F. Grützmacher and R. Asche, Chem. Ber, 108, 2080 (1975).

${ }^{3}$ F. J. Winkler and A. V. Robertson, Ibid. 109, 619 (1976).

${ }^{6}$ G. A. R. Kon and M. Q. i-Khuda, J. Chem. Soc. 3071 (1926).

${ }^{7}$ Massenspektrometrie (Edited by H. Kienitz) Kap. C3, S. 297 ff.

Verlag Chemie GmbH, Weinheim/Bergstrasse (1968).
${ }^{8}$ H. Feltkamp and W. Kraus, Liebigs Ann. 683, 74 (1965).

'W. Hūckel and Y. Riad, Ibid. 637, 33 (1960).

${ }^{10}$ S. Meyerson and A. Weitkamp, Org. Mass. Spectrom. 2, 603 (1969).

'T. H. Morton and L. Beauchamp, J. Am. Chem. Soc. 94, 3671 (1972).

${ }^{12} \mathrm{H}$. Budzikiewicz, C. Djerassi and D. H. Williams, Mass Spectrometry of Organic Compounds Kap. 2.2, S.107ff. HoldenDay, San Francisco (1967).

${ }^{13}$ R. H. Martin, F. W. Lampe and R. W. Taft, J. Am. Chem. Soc. 88, 1353 (1966).

${ }^{14}$ E. L. Eliel, Stereochemie der Kohlenstoffuerbindungen. Verlag Chemie GmbH, Weinheim/Bergstrasse (1966).

13L. Birkhofer, A. Ritter and F. Bentz, Chem. Ber. 97, $21 \%$ (1964).

${ }^{16} \mathrm{M}$. Neumann and W. S. Johnson, Org. Synth. 41, 9 (1961).

${ }^{17}$ J. M. Bec, J. Durand and J. Huet, Bull. Soc. Chim. Fr. 4344 (1972).

${ }^{18}$ Organikum, Organisch-Chemisches Grundpraktikum S. 489490. VEB Deutscher Verlag der Wissenschaften, Berlin (1974). 